\title{
On the consideration of the channel angle effects on the electromagnetic fields associated with inclined lightning channel
}

\begin{abstract}
In this study, the channel angle effects on the different electromagnetic fields components associated with inclined lightning channel are considered. Furthermore, the effect of channel angle and also observation point angle on different electromagnetic fields are compared to the corresponding fields associated with vertical lightning channel. The results show the channel angle is more effective on the vertical electric field compared to other field components while the vertical electric field has direct relationship with lightning induced voltage for some coupling models.
\end{abstract}

Keyword: Lightning; Electromagnetic fields; Return stroke current; Inclined channel 